\title{
Bacteria and Antibiotic Susceptibility Patterns of Cerebrospinal Fluid Isolated in Children with Bacterial Meningitis at Sanglah Hospital, Bali from 2016 to 2018
}

\author{
Gina Henny Kristianti ${ }^{*}$ I Gusti Ngurah Made Suwarba, Dewi Sutriani Mahalini, \\ I Wayan Gustawan, I Made Gede Dwi Lingga Utama
}

Department of Child Health, Medical Faculty of Udayana University, Denpasar, Indonesia

Email address:

gina.henny91@gmail.com (G. H. Kristianti)

${ }^{*}$ Corresponding author

\section{To cite this article:}

Gina Henny Kristianti, I Gusti Ngurah Made Suwarba, Dewi Sutriani Mahalini, I Wayan Gustawan, I Made Gede Dwi Lingga Utama. Bacteria and Antibiotic Susceptibility Patterns of Cerebrospinal Fluid Isolated in Children with Bacterial Meningitis at Sanglah Hospital, Bali from 2016 to 2018. Clinical Neurology and Neuroscience. Vol. 4, No. 3, 2020, pp. 51-56. doi: 10.11648/j.cnn.20200403.12

Received: June 22, 2020; Accepted: July 14, 2020; Published: July 28, 2020

\begin{abstract}
Periodic review from laboratory about the pathogens causing the bacterial meningitis and its antimicrobial sensitivity test result is important to determine the most common etiology of meningitis, since they can be varied according to time, geography, and patient's age. This study is a retrospective descriptive study. Affordable population in this study was children with positive cerebrospinal fluid culture results who were treated in Pediatric ward at Sanglah hospital, Denpasar from January 1st, 2016 until December 31st, 2018. The study used secondary data from the microbiology register in Clinical Microbiology Laboratory and medical record of Sanglah Hospital. This study employed total sampling method to collect samples. There were 52 samples that obtained in this study. Most of them were gram negative bacteria (53.8\%). The most common gram negative bacteria found were Pseudomonas sp, with Pseudomonas stutzeri. The most common gram positive bacteria found were Staphylococcus sp, with Staphylococcus hemolitikus. Vancomycin, Linezolid and Tigecyclin have high sensitivity against gram positive bacteria. Carbapenem, aminoglycoside and fourth generation of cephalosporine still had high sensitivity against gram negative bacteria. About $26.9 \%$ of bacterias were multidrug resistant organisms (MDRO) and most of them (78.6\%) were gram positive. Ceftriaxone as our empirical therapy has low sensitivity $(30 \%)$ to gram positive bacteria and moderate sensitivity to gram negative bacteria (53.57\%). Resistance to cephalosporin might be quite high in this particular clinical setting due to high number of MDRO. It should be taken into account before giving treatment for bacterial meningitis.
\end{abstract}

Keywords: Bacterial Meningitis, Culture Sensitivity, Children

\section{Introduction}

Acute bacterial meningitis is one of the most serious infections in infants and older children, it is among the 10 major causes of mortality from infectious disease all over the world. High incidence of acute complications and risk of long-term morbidity is associated with this disease. $[1,2]$. Despite advances in antibiotic therapy and vaccination strategies, children are particularly vulnerable to bacterial meningitis because of their relatively immature immune systems. [3]

It is estimated that over $75 \%$ of all cases of bacterial meningitis occur in children under 5 years of age. The WHO estimates that about 170.000 deaths occur annually from the disease worldwide; the case fatality rate can be as high as $50 \%$ if not treated. [3] About 5 to $10 \%$ of patients die within the first 24 hour of onset of symptoms in the absence of any treatment and around $10-20 \%$ of those who survived present severe neurological sequels such as hearing loss, learning disabilities, mental retardation, paralysis, and seizures. [4, 5]

Bacterial meningitis is most commonly caused by one of three types bacteria: Haemophilus influenzae type $b$ (Hib), Neisseria meningitidis, and Streptococcus pneumoniae bacteria which account for $90 \%$ of reported cases of acute 
bacterial meningitis in infants and children $>4$ weeks of age. Before the $1990 \mathrm{~s}, H$. influenzae type $b$ was the leading cause of bacterial meningitis but the new vaccine regimens, the occurrence of serious Hib disease have been reduced. [5] N. meningitidis is now considered to be the leading cause of bacterial meningitis in many regions of the world, causing an estimated 1.2 million cases of bacterial meningitis and sepsis worldwide each year. [6] The diagnosis is usually made by a lumbal puncture that demonstrates a turbid first drop of cerebrospinal fluid, a low glucose concentration, a high protein and neutrophil concentration with or without pleocytosis. Identification of the type of bacterial can be done either by gram stain, culture or by polymerase chain reaction (PCR) techniques. [5]

However, many infectious diseases are becoming increasingly difficult to treat because of antimicrobial resistant organisms. Both the epidemiology of bacterial meningitis and the sensitivity to antibiotics are changing as a result of the widespread use of antimicrobials and other factors. Periodic review from laboratory about the pathogens causing the bacterial meningitis and its antimicrobial sensitivity test result is important to determine the most common etiology of meningitis, since they can be varied according to time, geography, and patients age. It is also necessary to improve the clinical management of cases, guiding therapeutic managements so that the most appropriate treatment can be given timely. Further, it can assist in planning new preventive strategies. [1, 2] This study aims to determine the different bacteria causing meningitis and their antibiotic susceptibility pattern in tertiary hospital.

\section{Materials and Methods}

This study is a retrospective descriptive study, conducted in Pediatric ward at Sanglah hospital in March 2019. Target population in this study was children with positive Cerebrospinal Spinal Fluid (CSF) culture results who were treated in Pediatric ward at Sanglah hospital, Denpasar. Affordable population were children with positive CSF culture results who were treated in Pediatric ward at Sanglah hospital, Denpasar period 2016-2018.

Inclusion criterias in this study were patients aged 1 month until 18 years old with positive cerebrospinal fluid culture results from lumbal puncture with bacterial meningitis symptoms from January 1st 2016 until December 31st 2018. The exclusion criteria was patients who were treated with incomplete medical records.

Sample size were recruited using total sampling. Samples were obtained from the microbiology register in Clinical Microbiology Laboratory at Sanglah hospital and medical records from January 1st 2016 until December 31st 2018. Variables in this study are gender, age, examination period, shunt device, multi drug resistance organism (MDRO) and sensitivity test antibiotic. Age is age of patient by date, month, and year of birth seen from identity data on medical record. Gender is difference in sex that distinguish between men and women, the data presented in the form of categorical scale that is male and female. Cerebrospinal fluid (CSF) shunt device is a medical device installed to relieve pressure in the brain such as VP (Ventriculoperitoneal) shunt or EVD (External Ventricular Drain). Multidrug resistance organism is organism which is resistant to $\geq 1$ agent in $\geq 3$ antimicrobial categories. Antibiotic sensitivity test is antibiotic sensitivity examination using the disc diffusion method in order to see the growth of bacteria despite antibiotic administration. The data presented in the form of categorical scale that is using device shunt and not using device shunt. Examination period is the duration needed for bacterial growth in culture media until the culture results are released and expressed in days. The data presented in the form of categorical scale, which is less than or equal to 5 days and more than 5 days.

Protocol of this study are all the patients aged 1 month to 18 years, attending the Pediatric department who have done CSF analytic with positive culture result, were included in the study. The recording was made on a sample of research including common characteristics such as name, age, gender, shunt device, examination period and liquid cerebrospinal cultures following the test results of germ sensitivity to antibiotics. Data were obtained from the microbiology register in Clinical Microbiology Laboratory of Sanglah Hospital. Data were collected from January 1st, 2016 until December 31st, 2018.

Calculation of the highest proportions of bacteria and antibiotic sensitivity was calculated from the recording of the results of liquid cerebrospinal culture and sensitivity test. The collected data are presented in descriptive form of narrative and tables are equipped with frequency display and presentation.

This study has been approved by the Research Ethics Committee of the Faculty of Medicine, Udayana University/ Sanglah General Hospital. Study was conducted under the supervision of Pediatric Neurology Division, Medical Faculty of Udayana University.

\section{Result}

Between January 1st 2016 and December 31st 2018, there were 92 samples with positive culture results, 40 of them (43.4\%) were bacterial contaminated and 52 samples with bacterial meningitis. Of 52 samples collected, 24 samples (46.1\%) were gram positive bacteria and 28 samples (53.8\%) were gram negative bacteria. Twenty five samples were male and 27 samples $(52 \%)$ were female. Thirty two samples were 4 years old or younger and $20(38.5 \%)$ samples were older. Out of 52 samples with positive culture, 15 samples (28.8\%) were using devices such as VP shunt or EVD, and 37 samples were not. Most of Examination period (73.1\%) were less than 5 days. In our study about $26.9 \%$ bacterial were MDRO, which $11(78.6 \%)$ of the bacteria were gram positive.

The most common gram positive bacteria isolated was from Staphylococcus sp family, with Staphylococcus hemolitikus as dominant agent. The most common gram negative bacteria isolated was from Pseudomonas sp family, with Pseudomonas stutzeri as dominant agent. Data was presented in table below (Table 2). 
Table 1. Sample Characteristics.

\begin{tabular}{ll}
\hline Characteristics & $\mathbf{n}(\mathbf{\%})$ \\
\hline Gender & \\
Male & $25(48 \%)$ \\
Female & $27(52 \%)$ \\
Age & \\
1 month- 4 years old & $32(61.5 \%)$ \\
$>4$ years old & $20(38.5 \%)$ \\
Gram Staining & \\
Positive gram & $24(46.1 \%)$ \\
Negative gram & $28(53.8 \%)$ \\
Shunt device & \\
Yes & $15(28.8 \%)$ \\
No & $37(71.1 \%)$ \\
Examination periods & \\
$\leq 5$ days & $14(26.9 \%)$ \\
$>5$ days & $38(72.1 \%)$ \\
Total & $52(100 \%)$ \\
\hline
\end{tabular}

Table 2. Children Cerebrospinal Fluid (CSF) Culture Characteristics.

\begin{tabular}{ll}
\hline Bacterial type & (n)\% \\
\hline Gram-positive bacteries & 5 \\
Staphylococcus hemolitikus & 3 \\
Staphylococcus hominis & 3 \\
Staphylococcus cohnii & 3 \\
Staphylococcus Epidermidis & 2 \\
Streptococcus pneumonia & 2 \\
Enterococcus sp & 2 \\
Streptococcus viridans & 1 \\
Staphylococcus aureus & 1 \\
Staphylococcus warneri & 1 \\
Staphylococcus Saprophyticus & 1 \\
Staphylococcus capitis & \\
Gram-negative bacteries & 9 \\
Pseudomonas stutzeri & 6 \\
Eschericia colli & 4 \\
Acinetobacter Baumanii & 3 \\
Acinetobacter iwofii & 2 \\
Pseudomonas aeruginosa & 1 \\
Pseudomonas fluorescens & 1 \\
Acinetobacter junii & 1 \\
Enterobacter cloacae & 1 \\
Klebsiella oxytoca & 52 \\
Total
\end{tabular}

Antibiotic sensitivity pattern against positive gram bacteria were shown in the table below. Vancomycin, Linezolid dan Tigecyclin had high sensitivity against gram positive bacteria. Meanwhile, ceftriaxone had 30\% sensitivity, 54\% resistance against gram positive bacteria but $16 \%$ of the data was not available.

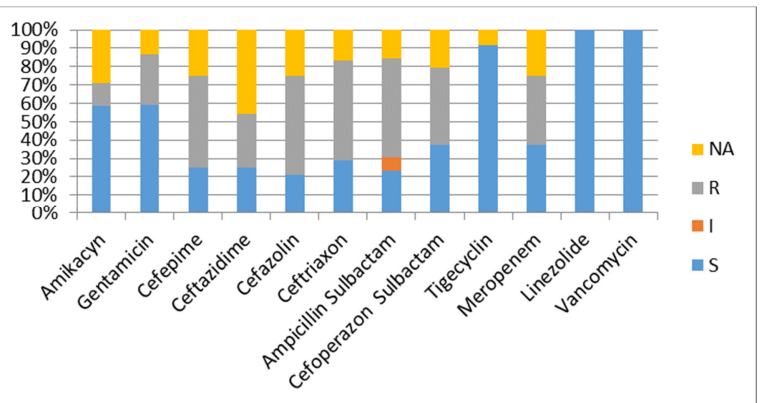

Figure 1. Antibiotic Susceptibility Pattern of Gram Positive Bacterial in Meningitis Children.
Antibiotics sensitivity pattern against gram negative bacteria was shown in the table below. Tigecyclin, Meropenem, Amikacin and Ceftazidime still had high sensitivity against them. Meanwhile ceftriaxone had 55.6\% sensitivity and $11.1 \%$ of them was resistant. However, $33.3 \%$ of the data was not available.

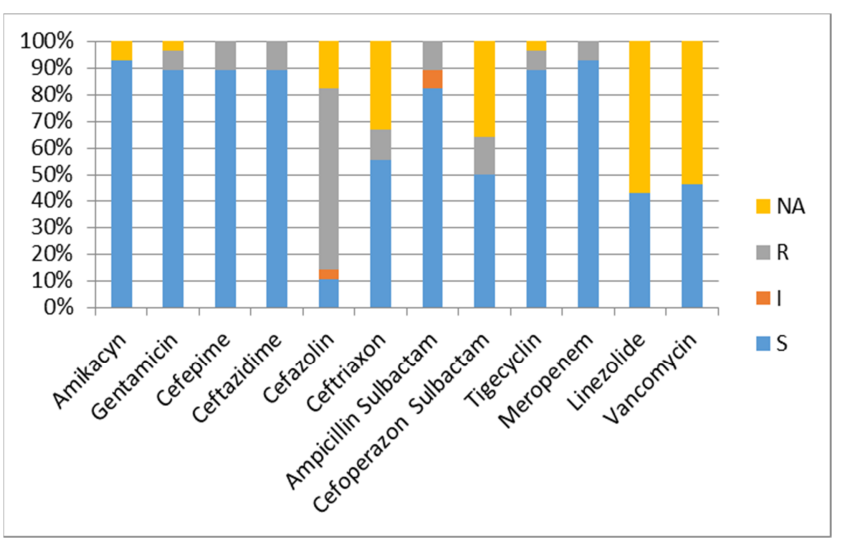

Figure 2. Antibiotic Susceptibility Pattern of Gram Negative Bacterial in Meningitis Children.

\section{Discussion}

Bacterial meningitis is a serious disease associated with high morbidity and mortality if the etiologic diagnosis is delayed. Since some common pathogens are labile (Neisseria meningitidis, Streptococcus pneumoniae), specimens of cerebrospinal fluid should be processed immediately after they are collected. The specimen must be collected aseptically and delivered immediately to the laboratory. It should not be refrigerated or exposed to extreme cold or excessive heat. They should be transported at temperatures between 20 degree Celsius and 35 degree Celsius. For proper culture results, CSF specimens must be plated within one hour. [7] In this study, there were 92 samples with positive CSF culture results during 2016-2018, and almost half were contaminants, only 52 samples were confirmed bacterial meningitis. The presence of a large number of contaminant germs were influenced by various factors. The way of taking and transporting samples (pre-analytic), as well as storing culture material for too long in the wrong place could affect the germ growth results. This result didn't reflect the actual pathogens. The fact that most patients received antimicrobial agents that are readily purchased as over the counter medications even prior to clinical evaluation alongside the occasional delay in CSF sampling, resulting in germ patterns obtained that do not reflect the real result. [8] Besides, according to Brouwer et al, CSF culture is documented to be positive only in $1 / 10$ of the previously antibiotic treated patients in developing countries. [9] Same result was found by Afifi et al who reported low rates of culture positive CSF samples ( $8 \%$ ) of suspected cases with bacterial meningitis. [10]

While the CSF culture is the gold standard in diagnosing bacterial meningitis, the low bacterial growth rates particularly in patients who have received antibiotic treatment 
before the lumbar puncture (LP) necessitated the development of new test methods. Nucleic acid amplification tests such as the polymerase chain reaction (PCR) can detect small amounts of pathogen DNA independently from the growth of the microorganism causing the disease. [8] Wang et al also identified bacterial meningitis in five cases $(9 \%)$ by CSF cultures and $25(45 \%)$ by real-time PCR. They considered real-time PCR much more sensitive than culture for the diagnosis of bacterial meningitis particularly in their study where $68 \%$ of patients had received prior antimicrobial treatment and their CSF samples yielded negative culture results. [11] A similar conclusion has been reached by Wu et al who stated that realtime PCR increases diagnostic yield for bacterial meningitis and is ideal for incorporation into routine surveillance in developing countries. [12]

In this study, the proportion of boys and girls with bacterial meningitis were almost the same. Study by Shrestha, et al also revealed similar result, in their study $55.2 \%$ were males and $44.8 \%$ were females, male and female showed equal distribution of culture positive cases. [13] Other study also showed that the proportion of meningitis in men and women is almost the same. These results showed no sex significant difference with male infection higher than females. [14]

Most of the cases of bacterial meningitis as evidenced by positive CSF culture are less than or equal to 4 years. Based on the study by Kather et al, it was found that more infection were in the age group of 1-month-two years with frequency (44\%), and $81 \%$ of confirmed bacterial meningitis belonged to age group 1-month-4 years, these age groups for the children have considered as development age and they are more susceptible to infection than elder one. [14]

In this study, gram negative bacteria were found more than gram positive bacteria. Mohammadi et al., reported that $73.33 \%$ gram negative and $26.67 \%$ gram positives had been isolated in their study, which were similar to our findings. [15]

Out of 52 samples with positive culture of bacterial meningitis, $15(28.8 \%)$ samples were using shunt device. Shunt infection is generally defined as the identification of a bacterial pathogen from the CSF both by gram stain and culture, in conjunction with CSF pleocytosis, fever, neurologic symptoms, and signs of shunt malfunction. Shunt infection rates per patient range from $10 \%$ to $22 \%$ and around $6.0 \%$ per procedure, with $90 \%$ of infections occurring within 30 days of surgery. These events are mostly attributable to normal skin flora such as coagulase-negative staphylococci, $S$. aureus and Propionibacter acnes, which are thought to be introduced at the time of surgery. Gram-negative organisms and Candida species have also been reported in CSF culture. Shunt infections are associated with higher rates of revision, recurrence of infection, ventriculitis, meningitis, and encephalitis, and often with greater mortality rates. [16]

In this study, the duration of culture examination result was almost less than 5 days. Desai et al found in 158 positive CSF specimens, approximately $25 \%$ of these grew organisms after $>$ 3 days, with some as long as 10 days after specimens were obtained. The most common organisms grown from individual patients were coagulase-negative Staphylococcus spp, Propionibacterium spp, Bacillus spp, Pseudomonas aeruginosa, and Staphylococcus aureus. Mean and maximum days to growth were different across species, with $S$. aureus showing the shortest and Propionibacterium spp showing the longest incubation times. Thus, a routine 10 day observation period for CSF specimens can be justified. [17, 18]

The epidemiology of acute bacterial meningitis has changed markedly since the introduction of conjugate vaccines. However, the disease continues to cause a heavy burden even in developed countries and causing substantial morbidity and mortality. Early administration of antibiotics save lives, but the globally emerging multidrug resistant bacteria limits the effectiveness of many inexpensive and widely available antimicrobial drugs. The molecular mechanisms of antibiotics resistance have been studied extensively and involved studying genetics and biochemical aspects of bacterial cell function. However, most of these mechanisms can be disseminated by one or more distinctive gene transfer mechanisms. Under selective pressure of certain antibiotics, bacterial variants evolve various mechanisms to survive in the presence of these antimicrobial agents. Drug resistant bacteria are usually multi-drug resistant against various structurally different drugs. Although antimicrobial resistance is classically attributed to chromosomal mutations, resistance is mainly associated with extra-chromosomal elements acquired from other bacteria in the environment, such as plasmids. [19, 20] In this study, about $26.7 \%$ of organisms were MDRO and most of them (78.6\%) were gram positive bacteria. Study in Egypt showed the similar result, out of the 71 isolates, 26 isolates $(36.6 \%)$ were MDR isolates. [19]

A study conducted in Europe and Mediterranean region during 2007 found that Neisseria meningitidis, Streptococcus pneumoniae and Haemophilus influenzae type $b$ were most commonly associated with bacterial meningitis accounting for almost $90 \%$ of reported cases of acute bacterial meningitis in infants over 60 days of age and young children. In contrast, another study was carried out in eastern of Mediterranean region during 2005-2010 found that the most commonly isolated pathogens were $S$. pneumoniae (27\% of confirmed cases), N. meningitidis (22\%), and H. influenzae (10\%). [19] Meanwhile in this study found the most common gram positive bacteria isolated was from Staphylococcus sp family, with Staphylococcus hemolitikus as dominant agent. The most common gram negative bacteria isolated was from Pseudomonas sp family, with Pseudomonas stutzeri as dominant agent.

The cases caused by staphylococci are usually secondary and may started as nosocomial infection after surgical intervention (most often neurosurgical) or as community-acquired disease after serious accompanying illnesses endocarditis, osteomyelitis, arthritis, otitis etc. Coagulase-negative staphylococci (CoNS) are normal inhabitants of the human skin and mucous membranes. Patients at highest risk for CoNS infection frequently have a disruption in their host defense mechanisms due to surgery, foreign body placement, or immunosuppression. Since CoNS 
are common contaminants of cultures, the diagnostic definition of CoNS meningitis should be defined with more strict criteria. Former study revealed that CoNS meningitis accounted for $11 \%(14 / 127)$ of bacterial meningitis and $19.4 \%$ (14/72) of postneurosurgical bacterial meningitis caused by a single pathogen. Of the 14 cultured CoNS strains, $S$. epidermidis was the most common subtype, accounting for $71 \%(10 / 14)$, and the other subtype, S. haemolyticus, accounted for $29 \%(4 / 14)$. [21] Pseudomonas stutzeri is an aerobic, nonfermenting, active, gram-negative oxidase-positive bacteria. It is mostly a saprophyte found in soil, water, Cases of P. stutzeri infection concern typically immunocompromised patients with underlying diseases or previous surgery. The most common reported sites of clinical isolates are surgical wounds, blood, respiratory tract, and urine. [22]

During the last two decades, there were major advances in the understanding of the etiology and pathophysiology of bacterial meningitis. However, the major breakthrough was in the prevention of meningitis with the introduction of the Hib conjugate vaccine in the early 1980s. This resulted in a significant decrease in the incidence of bacterial meningitis in areas where routine vaccination of infants was instituted. In our study, $H$ influenza was not isolated as the causative agent in any case, and only 2 subjects with Streptococcus pneumonia that we found. indicating that introduction of Hib vaccination and Pneumococcal vaccination in to the immunization schedule has yielded good results. [23] Moreover, the fact that most patients received antimicrobial agents that are readily purchased as over the counter medications even prior to clinical evaluation alongside the occasional delay in CSF sampling, causing bacterial pattern identified different from actual causes.

In bacterial meningitis, the blood-brain barrier is becoming leaky by the opening of intercellular tight junctions of the vessel walls particularly within venules. Moreover, the CSF outflow resistance increases, leading to a moderate reduction of the CSF production and absorption rates, and the activity of P-gp can be inhibited by proinflammatory cytokines. These three mechanisms (increased drug entry into the CSF and delayed removal by a reduction of the CSF bulk flow and by the inhibition of the activity of efflux pumps) synergistically lead to increased CSF concentrations, particularly of drugs that do not enter the CSF readily in the absence of meningeal inflammation. In our study Vancomycin, Linezolid and Tigecycline have high sensitivity against positive gram bacterial. Carbapenem, aminoglycoside and fourth generation of cephalosporine still had high sensitivity against negative gram bacterial. Ceftriaxone as our empirical therapy in bacterial meningitis has low sensitivity $(30 \%)$ to gram positive bacterial and moderate sensitivity to gram negative bacterial (53.57\%). Overuse of antimicrobials and the resulting resistance is one of the issues threatening global public health. Low income countries are heavily affected by antibiotic resistance due to high rate of infectious diseases and fewer antibiotic options. Due to increasing resistance, cephalosporins are frequently identified as a particular target for evaluation of antibiotic stewardship. Ceftriaxone is one of the third generation cephalosporins that is commonly prescribed due to its low toxicity and high efficacy against a wide range of bacterias. However, in an antimicrobial surveillance done by the WHO on all its six regions, the resistance pattern of Escherichia coli (E. coli) to third generation cephalosporins was $50 \%$ or more in five regions. All six regions reported Klebsiella pneumonia (K. pneumonia) had developed resistance in $50 \%$ or more cases while three regions reported that Neisseria gonorrhoea had developed 25\% or more resistance to third generation cephalosporins $[13,24]$ Ceftriaxone is effective as monotherapy in children with meningitis caused by susceptible $S$. pneumonia, but in our study S. pneumonia was not the major etiology of meningitis. It is also potentially inadequate as empiric therapy of meningitis after neurosurgery because there is an increased likelihood of MRSA, $P$. aeruginosa and other ceftriaxone-resistant Gram-negative bacilli in this patient population. In this study, almost $30 \%$ of subject were using shunt device and about $26,9 \%$ of organisms that we found were MDRO. Another study in Egypt, revealed the similar result. Ceftriaxone has $61 \%$ of sensitivity and $34.3 \%$ of resistance to microbes in bacterial meningitis. Vancomycin has the highest sensitivity to microbes $(89.5 \%)$. [19]

Understanding the relationship between emerging bacterial resistance and antibiotic use requires monitoring of antibiotics. Furthermore, previous prescription of antibiotics leads to false negative results in culture and a decrease in the sensitivity of culture techniques, which may also affect our results. In this study, we investigated only culture-positive bacterial meningitis in children with bacterial meningitis symptoms and those with negative results in cultures were excluded, we didn't explain the number of negative cultures in bacterial meningitis children. Prospective study is needed to describe clearly about the characteristic of bacterial culture in bacterial meningitis children.

\section{Conclusions}

This study revealed that there were no difference in the prevalence of bacterial meningitis between male or female. More gram negative than gram positive as etiology of bacterial meningitis with most of examination periods were less than 5 days. The incidence of bacterial meningitis is more common in young children. In this study found the most common gram positive bacteria isolated was from Staphylococcus sp, with Staphylococcus hemolitikus as dominant agent. The most common gram negative bacteria isolated was Pseudomonas sp, with Pseudomonas stutzeri as dominant agent. Vancomycin, Linezolid and Tigecycline have high sensitivity against gram positive bacterial. Carbapenem, aminoglycoside and fourth generation of cephalosporine still had high sensitivity against gram negative bacterial. Resistance to beta lactams and cephalosporin might be quite high in this particular clinical setting due to high number of MDRO. It should be taken into account in the future before administering therapy in bacterial meningitis cases. 


\section{Disclosure}

All the authors do not have any possible conflicts of interest.

\section{References}

[1] Chugh Y, Kastury N, Kapoor AK. Study of antimicrobial sensitivity pattern of Gram-positive CSF isolates among children suffering from septic meningitis in a tertiary care hospital. Journal, Indian Academy of Clinical Medicine. 2011. 12 (4): 274-82.

[2] Sudharshan R, Reddy P, Neelima A. Pattern and antibiogram of bacterial meningitis in children at a tertiary care hospital. Journal of Scientific and Innovative Research. 2013; 2 (6): 1012-6.

[3] Agrawal, S. Nadel, S. Acute Bacterial Meningitis in Infants and Children. Pediatr Drugs. 2011; 13 (6): 385-400.

[4] Karou SD, Balaka A, Bamoke M, Tchelougou D, Assih M, Anani K, Agbonoko, Simpore J, de Souza C. Epidemiology and antibiotic resistance of bacterial meningitis in Dapaong, northern Togo. Asian Pac J Trop Med. 2012; 5 (11): 848-52.

[5] Eldin K, Nageeb E, Saeed M, Elsayeb AA, Ibrahim SA. Pattern of bacterial meningitis in Sudanese children, Omdurman, Sudan. African journal of microbiology research. 2010; 4 (24): 2670-3.

[6] Ceyhan M, Yidirim I, Balmer P, Borrow R, Dikici B, Turgut M, et al. 2008. A Prospective Study of Etiology of Childhood Acute Bacterial Meningitis, Turkey. Emerg Infect Dis. 2008; 14 (7): 1089-96.

[7] Kim BN, Peri AM, Paterson DL. Ceftriaxon. In: Grayson ML, editor. Kucer's The Use of Antibiotics $7^{\text {th }}$ ed. Boca Raton: CRC Press; 2017. p. 464-529.

[8] Başpınar EO, Dayan S, Bekçibaşı M, Tekin R, Ayaz C, Deveci Ö, Hoşoğlu S. Comparison of culture and PCR methods in the diagnosis of bacterial meningitis. Brazilian Journal of Microbiology. 2017: 48 (2); 232-6.

[9] Brouwer MC, Tunkel AR, Van De Beek D. Epidemiology, diagnosis, and antimicrobial treatment of acute bacterial meningitis. Clinical Microbiology Reviews. 2010; 23 (3): 467-92.

[10] Afifi S, Wasfy MO, Azab MA. Laboratory-based surveillance of patients with bacterial meningitis in Egypt (1998-2004). European Journal of Clinical Microbiology and Infectious Diseases. 2007; 26 (5): 331-40.

[11] Wang Y, Guo G, Wang H. Comparative study of bacteriological culture and real imefluorescence quantitative PCR (RT-PCR) and multiplex PCR- ased reverse line blot (mPCR/RLB) hybridization assay in the diagnosis of bacterial neonatal meningitis. BMCPediatr. 2014; 14: 224-30.

[12] Wu SM, Cordeiro BH, Harcourt. Accuracy of real-time PCR, Gram stain and culture for Streptococcus pneumoniae,
Neisseriameningitidis and Haemophilus influenza meningitis diagnosis. BMC Infectious Diseases. 2013; 13 (1): 26.

[13] Shrestha RG, Tandukar S, Ansari S, Subedi A, Shrestha A, Poudel R, Sherchand JB. Bacterial meningitis in children under 15 years of age in Nepal. BMC Pediatrics. 2015; 15 (1): 1-7.

[14] Khater WS, Elabd SH. Identification of Common Bacterial Pathogens Causing Meningitis in Culture-Negative Cerebrospinal Fluid Samples Using Real-Time Polymerase Chain Reaction. International Journal of Microbiology, 2016, 17-19.

[15] Mohammadi SF, Patil AB, Nadagir SD, Nandhihal N, Lakshminarnyana SA. Diagnostic value of latex agglutination test in diagnosis of acute bacterial meningitis. Ann Indian Acad Neurol. 2013; 16 (4): 645-9.

[16] Mengistu A, Gaeseb J, Uaaka G, Ndjavera C, Kambyambya K, Indongo L, Sagwa E. Antimicrobial sensitivity patterns of cerebrospinal fluid (CSF) isolates in Namibia: Implications for empirical antibiotic treatment of meningitis. Journal of Pharmaceutical Policy and Practice. 2013; 6 (1): 1.

[17] Murgas YG, Snowden JN. Ventricular shunt infections: Immunopathogenesis and clilnical management. J Neuroimmunol. 2014; 276 (0): 1-8.

[18] Desai A, Lollis SS, Missios S, Radwan T, Zuaro DE, Schwarzman JD, Duhaime AC. How long should cerebrospinal fluid cultures be held to detect shunt infections? Clinical article. Journal of Neurosurgery: Pediatrics, 2009: 4 (2); 184-9.

[19] Abdelkader MM, Aboshanab KM, El-Ashry MA, Aboulwafa MM. Prevalence of MDR pathogens of bacterial meningitis in Egypt and new synergistic antibiotic combinations. PLoS ONE, 2017; 12 (2): 1-20.

[20] Jarousha AM, Afifi A. Epidemiology and risk factors associated with developing bacterial meningitis among children in Gaza strip. Iranian Journal of Public Health. 2014; 43 (9): 1176-83.

[21] Huang CR, Lu CH, Wu JJ, Chang HW, Chien CC, Lei CB, Chang WN. Coagulase-negative staphylococcal meningitis in adults: Clinical characteristics and therapeutic outcomes. Infection. 2005; 33 (2): 56-60.

[22] Bisharat N, Gorlachev T, Keness Y. 10-years hospital experience in Pseudomonas stutzeri and literature review. Open Infectious Diseases Journal. 2012; 6 (1): 21-24.

[23] Thomas V, Riaz Ahmed S, Qasim S. Cerebro spinal fluid analysis in childhood bacterial meningitis. Oman Medical Journal. 2008; 23 (1): 32-3.

[24] Berhe YH, Amaha ND, Ghebrenegus AS. Evaluation of ceftriaxone use in the medical ward of Halibet National Referral and teaching hospital in 2017 in Asmara, Eritrea: A cross sectional retrospective study. BMC Infectious Diseases. 2019: 19 (1), 1-7. 